\title{
Assistance au suicide et «fatigués de la vie»
}

Une étude présentée récemment par l'Institut de médecine légale de Zurich portant sur la ville de Zurich (ses conclusions ne peuvent donc pas sans autre être étendues à tout le pays) montre que ce ne sont pas seulement des malades en grande souffrance et à peu de temps de leur décès naturel qui font appel aux organisations d'aide au suicide [1]. Il y a aussi, de plus en plus semble$\mathrm{t}$-il, des personnes présentant des affections dont le pronostic n'est pas funeste à court terme et pas toujours très invalidantes. Cette situation pose à l'évidence question.

Comme une majorité de nos concitoyens (à en croire plusieurs sondages), je suis attaché à la liberté personnelle de décider de mettre un terme à sa vie, souvent une vie longue, quand celle-ci devient trop lourde à porter à cause d'une santé irréversiblement et gravement altérée. Toutefois, cela ne signifie en aucune manière que ce serait «bien» d'envisager le suicide, que ce serait une option comme les autres. En particulier, même si l'article 115 de notre Code pénal dispose que l'assistance au suicide n'est pas punissable sauf si elle est motivée par des mobiles égoïstes, les pouvoirs publics doivent éviter toute attitude donnant l'impression qu'ils cautionneraient le suicide; c'est dire qu'ils sont concernés quand l'aide au suicide est offerte à des personnes «simplement» fatiguées de la vie.

Rappelons que, bien sûr, l'autorité publique et la santé publique ont un rôle de prévention du suicide; ce rôle est spécialement important s'agissant de personnes vulnérables, dépressives, isolées, précarisées, comme le sont souvent les «fatigués de la vie». Et on ne saurait prendre note sans autre du fait que ces derniers, dont l'existence n'est en soi pas menacée à court terme, font appel à l'assistance au suicide. A mon sens, pour des malades en fin de vie présentant une affection mortelle, l'activité de ces organisations est acceptable, ne pose pas de problème majeur; la Commission nationale d'éthique (CNE) a jugé qu'il n'y avait pas lieu de condamner (ni moralement ni déontologiquement) les médecins qui acceptent dans ce contexte de prescrire aux patients un produit à dose létale. Mais la situation paraît bien différente s'agissant de personnes qui décideraient d'en finir sur la base d'un mal-être du registre psychologique ou social, hors d'une situation pathologique grave et de souffrance majeure. Ethiquement, humainement et d'un point de vue de sens commun, se pose la question de savoir si on doit admettre que des dispositifs organisés dans ce but offrent à ces personnes de les aider. Sans préjudice au droit strict de chacun d'envisager le suicide, c'est le caractère organisé qui fâche ici, sans qu'il y ait comme dans les cas de maladie mortelle à court terme une (sorte de) relation patientsoignant. Ne peut-on craindre une glissade vers «l'usine à mourir»?

De l'avis de la CNE [2-4], une supervision publique des activités des organisations d'aide au suicide est nécessaire - supervision qui au demeurant n'est guère aisée à définir. Pour ma part, je ne voudrais pas d'un système où des déterminations personnelles, nominatives, de mettre fin à ses jours devraient préalablement être entérinées (étiquetées de raisonnable) par une autorité ou une commission publique (rappelons qu'il y a toujours un contrôle a posteriori des conditions de ces décès par un juge d'instruction). Il s'agirait plutôt, par le canal d'un devoir d'annonce des organisations concernées, de s'assurer qu'elles satisfont à des critères et conditions, et de pouvoir ainsi mieux apprécier leur fonctionnement - y compris en effectuant les contrôles nécessaires. Leur champ d'action devrait être restreint aux personnes en grave souffrance, médicalement sans espoir. On s'assurera aussi qu'elles n'en font pas une activité lucrative - ce qui vraisemblablement représenterait un mobile égoïste au sens du Code pénal et la rendrait punissable.

Comme toujours dans les affaires de la société, il y a lieu ici de définir les contours d'une tolérance, d'un juste milieu, tenant compte des libertés fondamentales de l'individu, des tâches que doivent assumer les pouvoirs publics, des valeurs de la communauté et de son intérêt à éviter des dérapages éthiquement et pratiquement problématiques.

Dr Jean Martin, ancien médecin cantonal, membre de la Commission nationale d'éthique

Dr Jean Martin,
ancien médecin cantonal,
membre de la Commission nationale d'éthique

d'assistance au suicide doivent être surveillées. Le Temps, 14 juin 2007, p.19. 